\title{
LETTER TO THE EDITOR TET2 stabilization by 14-3-3 binding to the phosphorylated Serine 99 is deregulated by mutations in cancer
}

\author{
Cell Research (2019) 29:248-250; https://doi.org/10.1038/s41422-018-0132-5
}

\section{Dear Editor,}

The Ten-Eleven Translocation-2 (TET2) gene encodes an enzyme that catalyzes the conversion of 5-methylcytosine $(5 \mathrm{mC})$ to 5 hydroxymethylcytosine $(5 \mathrm{hmC})$, the key initial step in active DNA demethylation., ${ }^{1,2}$ TET2 and $5 \mathrm{hmC}$ function cooperatively as epigenetic tumor suppressors and are frequently deregulated in cancer. ${ }^{2}$ We recently reported that TET2 is a bona fide substrate of the AMP-activated kinase (AMPK), which phosphorylates TET2 serine 99 (S99) at its non-catalytic N-terminal region. ${ }^{3}$ Under normal glucose conditions, this post-translational modification stabilizes the TET2 protein. Importantly, we have demonstrated that in hyperglycemic conditions, S99 phosphorylation is impaired, thereby destabilizing TET2 and inactivating its tumor suppressive function. ${ }^{3}$ These findings unveiled a "phospho-switch" that serves to regulate TET2 stability and identified a cellular pathway by which adverse extracellular signals, including high glucose, act to disable the "phospho-switch," resulting in increased cancer risk. However, the underlying molecular mechanisms through which the $\mathrm{S} 99$ phospho-switch protects TET2 stability and how this regulatory mechanism is targeted by cancer mutations are still unknown.

To date, more than 2000 TET2 mutations have been reported. ${ }^{4,5}$ Phosphorylation site-weighted Mutation Set Enrichment Analysis (MSEA) revealed that the S99-centered region (defined as S99 flanking $+/-10 a a$ ) is one of 14 potential mutation clusters (peaks 1-14) on TET2 (Fig. 1a, dashed green box, here-in called "S99 phospho-switch cluster". For detailed analysis and identification of phosphorylation sites-enriched TET2 mutation clusters, please see Supplementary information, Fig. S1a). We have previously reported that the S99-centered region (S99+/-10aa region) contains one of the AMPK phosphorylation recognition motifs on TET2. ${ }^{3}$ Motif analysis by Scansite revealed that the same region (S99 flanking + /-10aa) also possesses a highly conserved 14-3-3binding motif. In fact, S99 is the only putative 14-3-3 binding site identified on the TET2 protein by the Scansite analysis with highly stringent settings (Fig. 1a, green box; Supplementary information, Fig. S1b). We therefore postulated that the S99 phospho-switch is not only modulated by AMPK, but also a novel 14-3-3 recognition site that mediates the interaction between TET2 and 14-3-3 proteins (Fig. 1b).

To explore the member(s) of $14-3-3$ protein family that potentially interact with TET2, we purified the TET2 complex from HEK293T cells using the tandem-tagged purification (TAP) approach. Proteomic analysis of the TET2 complex identified previously reported TET2-interacting partners, such as OGT and HDAC1, as well as an abundance of 14-3-3Z/D and 14-3-3E proteins (Fig. 1c; Supplementary information, Table S1). ${ }^{6}$ This was further confirmed through immunoblotting with 14-3-3 specific antibodies (Supplementary information, Fig. S2a). We were unable to detect 14-3-3 family proteins in TET1 or TET3 complexes purified under the same experimental conditions (Fig. 1d and Data not shown). Endogenous Tet2/14-3-3 association was also confirmed using a Co-IP assay in mouse embryonic cells (Supplementary information, Fig. S2b). Moreover, TET2 was reciprocally co-immunoprecipitated by $14-3-3 Z / D$ and $14-3-3 E$ (Supplementary information, Fig. S2c). Importantly, we determined that the $\mathrm{N}$-terminus of TET2 is responsible for the specific interaction between TET2 and 14-3-3 (Supplementary information, Fig. S2d). In agreement with previous reports, we validated that a small portion of 14-3-3 proteins are present in the cell nucleus (Supplementary information, Fig. S3a, b). ${ }^{7}$ Importantly, we observed enriched co-localization of TET2 and nuclear 14-3-3 proteins after treatment with an AMPK activator, A769662 (Supplementary information, Fig. S3b, c). These data demonstrate that 14-3-3 family proteins interact with the N-terminus of TET2 and are previously unrecognized, integral components of the TET2 complex.

As 14-3-3 proteins are well-characterized phospho-reader proteins, ${ }^{8}$ we set out to determine whether $14-3-3$ is a TET2S99 phospho-reader protein. Shown in Supplementary information, Fig. S4a, activation of AMPK by metformin significantly increased the level of TET2pS99, and, concurrently, enhanced the interaction between TET2 and 14-3-3. Conversely, inhibition of AMPK by a well-characterized inhibitor, compound $C$, abolished the phosphorylation of TET2 on S99 and diminished the interaction between TET2 and 14-3-3 (Supplementary information, Fig. S4b). Consistent with these findings, knockdown of the a2 subunit of the AMPK holoenzyme dramatically reduced the level of TET2pS99. Consequently, the interaction between TET2 and 143-3 family proteins was also significantly reduced (Supplementary information, Fig. S4c). Using an in vitro phosphorylation-coupled binding assay with purified recombinant TET2 and 14-3-3 proteins, we demonstrated that the interaction between the two proteins is direct and solely dependent on AMPK-catalyzed TET2pS99 (Supplementary information, Fig. S4d). To quantitatively determine the direct interaction between TET2 and 14-3-3, we next used isothermal titration calorimetry (ITC) to measure the binding kinetics of the two partners. The ITC data showed that 14-3-3 preferentially binds to TET2pS99 peptides with a Kd of $0.81 \mu \mathrm{M}$, whereas there is no measurable $\mathrm{Kd}$ for binding to unmodified TET2S99 (Fig. 1e; Supplementary information, Fig. S5a-c; Supplementary information, Table S2). To confirm that TET2pS99 represents the major 14-3-3 interacting moiety on TET2, we asked whether a full-length TET2S99A mutant could still bind 14-3-3 in cells. Using Co-IP, we verified that the S99A mutation disrupted the interaction between TET2 and 14-3-3Z/D, whereas the association with OGT was unaltered (Fig. 1f). Collectively, these data demonstrate that $\mathrm{S} 99$ phosphorylation, catalyzed by AMPK, is necessary and sufficient to mediate the direct interaction between TET2 and 14-3-3.

Studies have suggested that a "phospho-switch" imparted by a specific phosphorylation site and 14-3-3 binding plays a critical role in regulating protein stability. ${ }^{8}$ To uncover the functional importance of $14-3-3$ in protecting the stability of TET2, we treated cells with two 
a

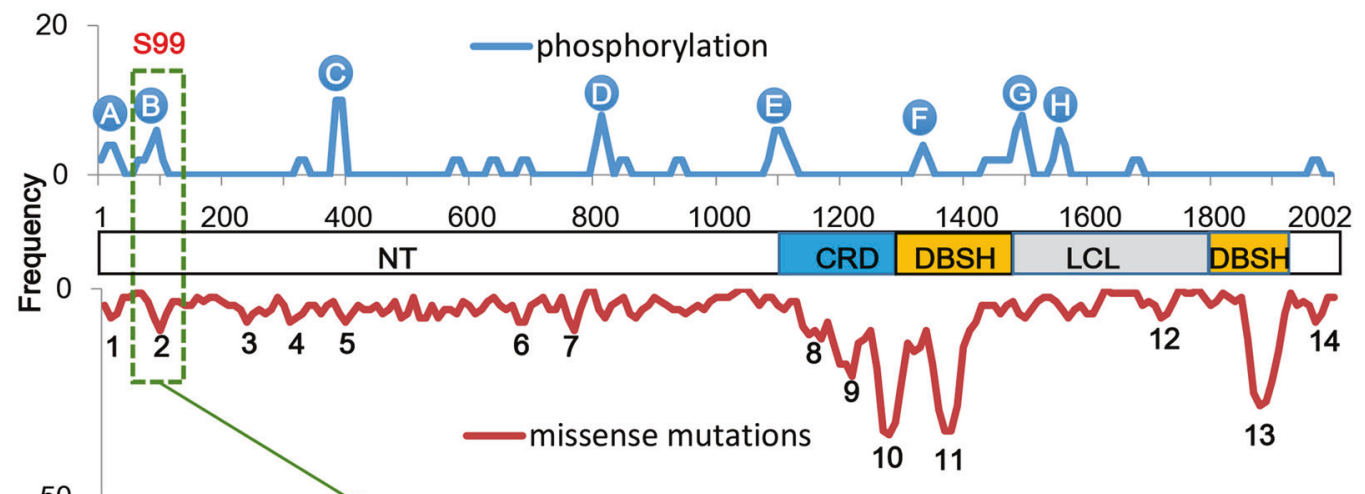

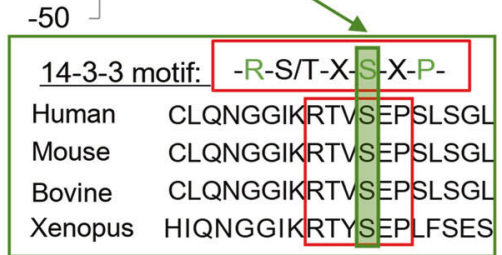

b

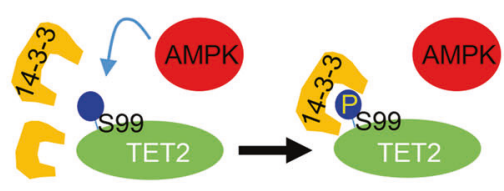

e $14-3-3 Z / D+$

1. LQNGGIKRTVS ${ }_{99} E$ PSLSG

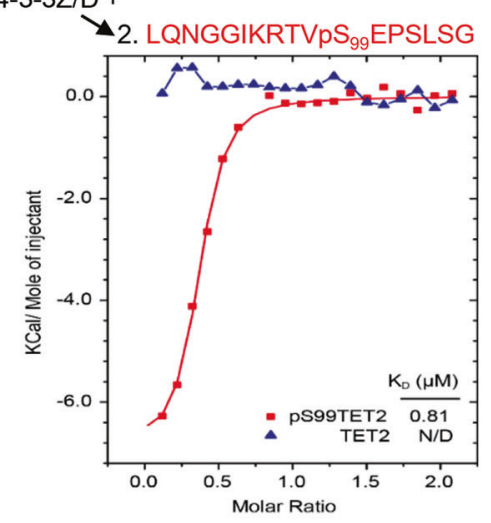

h

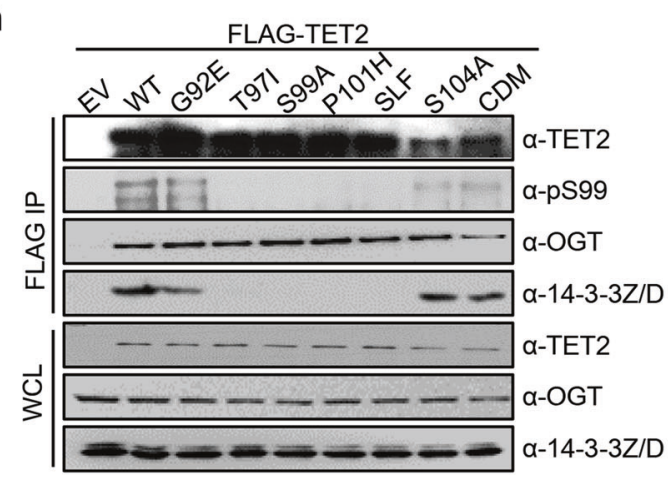

different 14-3-3 inhibitors that effectively disrupt interactions between 14-3-3 family proteins and their phosphorylated binding partners. ${ }^{9}$ Inhibitor treatment did not reduce S99 phosphorylation (Supplementary information, Fig. S5d). Nonetheless, we found that both inhibitors significantly reduced TET2WT levels in cells, with only a marginal
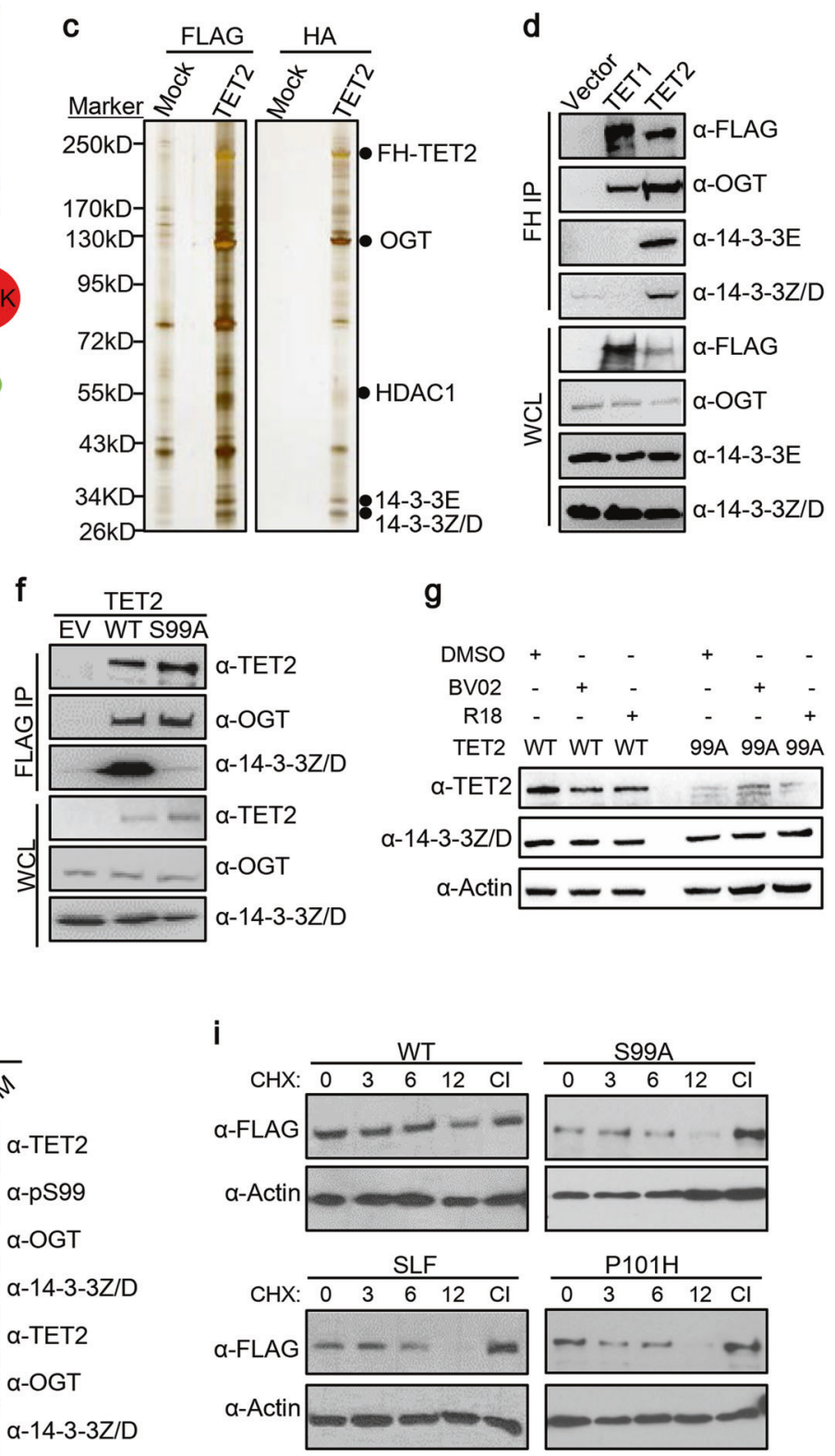

effect on the TET2S99A mutant protein, as it was not protected by 143-3 and its basal level was already very low (Fig. 1g). Consistently, TET2 protein level dramatically decreases after 14-3-3Z/D knockdown, suggesting that 14-3-3Z/D is critical for safeguarding the steady-state level of TET2 proteins (Supplementary information, Fig. S5e, f). 
Fig. 1 A cancer mutation cluster flanking TET2 Serine 99 disrupts its interaction with 14-3-3 and leads to TET2 destabilization. a A superimposed schematic map of phosphorylation-associated cancer mutation clusters on TET2. The blue line indicates 8 possible phosphorylation "islands" in TET2 (peaks a-h). The red line depicts 14 possible cancer mutation clusters spanning the TET2 coding region identified by conventional mutation enrichment analysis. The green dashed box indicates the S99 cluster. Conserved 14-3-3 binding motif was shown in a green box. b Diagram of possible model for TET2/14-3-3 interaction through the phosphorylated S99 modulated by AMPK. c Silver staining of the Flag:HA-tandem-purified TET2 complex and proteomic analysis of the TET2 complex. d Differential association of 14-3-3 with the TET1 and TET2 complexes. TET2, but not TET1 specifically interacts with 14-3-3 family members. e Differential kinetics of TET2S99 and TET2pS99 peptide binding to 14-3-3 was determined by ITC. f The TET2 S99A mutation abolishes the direct TET2/14-3-3 interaction. g Disruption of 14-3-3/TET2 interaction by 14-3-3 inhibitor treatment reduces TET2 protein level. $\mathbf{h}$ Characterization of the functional effect of the 599 cluster mutants on the association between TET2 and 14-3-3 proteins. i Half-life measurements indicate that mutations in the S99 cluster accelerate TET2 turn-over. Technical note: The steady state of TET2 would be impaired either by the S99A mutation or downregulation of AMPK as we have previously shown. ${ }^{3}$ To ensure that the greatly diminished level of 14-3-3/TET2 interaction is due to the mutation or lack of AMPK-mediated S99 phosphorylation, but not caused by the total protein level that was dramatically reduced, the cells used for Co-IP to examine the 14-3-3/TET2 interaction were additionally pre-treated with calpain inhibitors to protect TET 2 protein from degradation. To have a comparable amount of total WT and mutant TET2 to begin with for the co-IP, the DNA amounts of the mutant or WT TET2 expression vectors for transient transfection may also be adjusted according to the actual WT or mutant protein overexpressed. This technical note also applies to the Supplementary information, Fig. S4b, c

The "S99 phospho-switch cluster" harbors several recurrent mutations, including the reported G92E, R96C, T97I, P101H, P101T, S102_L103 > F (SLF), and L103V mutations (Supplementary information, Fig. S6a). We have identified that a few of the naturally mutated residues are involved in AMPK recognition and 14-3-3 binding. R96 and L103 are critical sites for AMPK recognition, while R96, T97, and P101 are believed to play a role in 14-3-3 binding. ${ }^{8,10}$ We created TET2 mutant proteins carrying these mutations and examined their stability and interaction with 14-3-3 in cells. Similar to TET2WT, the mutant proteins all localized to the nucleus (Supplementary information, Fig. S6b). However, when critical 14-3-3-binding sites, such as T97 and P101 were mutated, both $\mathrm{S} 99$ phosphorylation and the interaction between 14-3-3 and TET2 mutants were largely abolished. In contrast, mutations that did not disrupt AMPK and/or 14-3-3 recognition, such as G92E, S104A or the catalytic inactive mutant $\left(\mathrm{H}_{1382} \mathrm{RD}_{1384}>\right.$ $\mathrm{Y}_{1382} \mathrm{RA}_{1384}$ ), had little impact on $\mathrm{S} 99$ phosphorylation or the TET2/ 14-3-3 interaction (Fig. 1h). Moreover, the half-lives of 14-3-3 binding-defective mutants, such as S102_L103>F (SLF) and $\mathrm{P} 101 \mathrm{H}$, were shorter than that of the WT (Fig. 1i). Nevertheless, as predicted, when the cells were treated with calpain inhibitor, which reverts the instability of the S99A mutant protein as shown previously, the level of SLF and $\mathrm{P} 101 \mathrm{H}$ mutant proteins were significantly restored.

In summary, this study reports a novel finding that 14-3-3 proteins are TET2pS99 phospho-readers that bind to S99phosphorylated TET2 and are crucial in governing the TET2S99 phospho-switch. We propose that the interaction of 14-3-3 with TET2pS99 stabilizes the tumor suppressor and this stabilization is likely one of the underlying molecular mechanisms by which the TET2 protein is protected from the calpain protease-mediated degradation. TET2 mutations in the "S99 phospho-switch cluster", a region that is critical for 14-3-3 binding, abolish the interaction between the proteins, thereby promoting TET2 instability. Additionally, calpain inhibitor treatment reverts TET2 instability arising from mutations in the "S99 phospho-switch" cluster. Thus, this study lays the groundwork for future clinical investigation of cancer therapeutics targeting the $\mathbf{5 9 9}$ phospho-switch mutation cluster.

\section{ACKNOWLEDGEMENTS}

The authors would like to thank Barry Zee, Tobin Gramyk, George Murphy and Christine Lian for critical reading of the manuscript and insightful comments, Guoming Shi, Jia Fan, Fei Lan and Lingchun Kong for their help and advice in experimental design, Anna Payne-Tobin Jost and Jennifer Waters of NIC at Harvard for the supports in imaging, and Santa Cruz Biotechnology, Inc. for providing antibodies. This work was supported by NIH grants GM112062 and CA194302 to Y.G.S.

\section{AUTHOR CONTRIBUTIONS}

H.C. and Y.G.S. conceived and designed the study. H.C., D.Y., K.M., R.F., D.W. and D.H. performed most of the experiments. P.J. and Z.Z. carried out the MSEA analysis. Z.W. and J.P. were responsible for mass spectrometry determination. H.C. and Y.G.S. wrote the manuscript and all authors contributed to the final version of the manuscript. Y.S. and Y.G.S. supervised the project.

\section{ADDITIONAL INFORMATION}

Supplementary information accompanies this paper at https://doi.org/10.1038/ s41422-018-0132-5.

Competing interests: Y.S. is a co-founder of Constellation Pharmaceuticals and Athelas Therapeutics, and a consultant for Active Motif, Inc. Remaining authors declare no competing financial interests.

Publisher's note: Springer Nature remains neutral with regard to jurisdictional claims in published maps and institutional affiliations.

Hao Chen $\mathbb{D}^{1,2}$, Dingdang $\mathrm{Yu}^{1}$, Rui Fang ${ }^{3}$, Kimberlie Rabidou ${ }^{3}$, $\mathrm{Di} \mathrm{Wu}{ }^{3}, \mathrm{Di} \mathrm{Hu} \mathrm{DD}^{3}$, Peilin Jia ${ }^{4}$, Zhongming Zhao $\mathbb{D}^{4}$, Zhiping $\mathrm{Wu}^{5}$, Junmin Peng ${ }^{5}$, Yang Shi ${ }^{2}$ and Yujiang Geno Shi ${ }^{1,3}$

${ }^{1}$ Laboratory of Epigenetics, Liver Cancer Institute, Zhongshan Hospital, and Institutes of Biomedical Sciences, Fudan University,

Shanghai 200032, China; ${ }^{2}$ Division of Newborn Medicine and Program in Epigenetics, Children's Hospital, Harvard Medical School, 300 Longwood Avenue, Boston, MA 02115, USA; ${ }^{3}$ Endocrinology Division, Brigham and Women's Hospital, Harvard Medical School,

221 Longwood Avenue, Boston, MA 02115, USA; ${ }^{4}$ Center for Precision Health, School of Biomedical Informatics, The University of Texas Health Science Center at Houston, Houston, TX 77030, USA and ${ }^{5}$ Departments of Structural Biology and Developmental Neurobiology, St. Jude Children's Research Hospital, Memphis, TN 38105, USA

Correspondence: Yujiang Geno Shi (yujiang_shi@hms.harvard.edu)

\section{REFERENCES}

1. He, Y. F. et al. Science 333, 1303-1307 (2011)

2. Wu, X. \& Zhang, Y. Nat. Rev. Genet. 18, 517-534 (2017).

3. Wu, D. et al. Nature 559, 637-641 (2018).

4. Forbes, S. A. et al. Nucleic Acids Res. 45, D777-D783 (2017).

5. Desai, P. et al. Nat. Med. 24, 1015-1023 (2018).

6. Vella, P. et al. Mol. Cell 49, 645-656 (2013).

7. Esteve, P. O. et al. Nucleic Acids Res. 44, 1642-1656 (2016).

8. Morrison, D. K. Trends Cell Biol. 19, 16-23 (2009).

9. Zhao, J., Meyerkord, C. L., Du, Y., Khuri, F. R. \& Fu, H. Semin. Cell \& Dev. Biol. 22, 705-712 (2011).

10. Gwinn, D. M. et al. Mol. Cell 30, 214-226 (2008). 